\title{
The significance of velocity exponents in identifying erosion- corrosion mechanisms
}

\author{
M.M. Stack, F.H. Stott and G.C. Wood
}

University of Manchester Institute of Science and Technology, Corrosion and Protection Centre, P.O. Box 88, Sackville St., Manchester, M60 1QD, Great Britain

\begin{abstract}
The modes of erosion-corrosion are diverse and may vary from being "erosiondominated", where erosion of metal is the dominant process, to "corrosion-dominated", where erosion of oxide scale is the dominant process. The intermediate situation in which erosion of transient oxide is the predominant process is termed "erosion-corrosion-dominated" and describes the regime in which continual formation and removal of oxide occurs down to the scale/metal interface. This paper considers some of the recent erosion-corrosion data and evaluates the velocity exponents. The critical factors which affect velocity exponents in these environments are identified, and some general principles and provisos are outlined when attempting to use such a technique to identify the mechanism of erosion-corrosion on the material surface. It is shown that the velocity exponents derived for "erosion-dominated" conditions are similar to those evaluated for "ductile" erosion processes. However, for "corrosion-dominated" conditions the exponents are significantly lower than those derived for "brittle" erosion processes at room temperature. For "erosion-corrosion-dominated" conditions the situation is more complex with velocity exponents being strongly dependent on temperature, alloy composition and relationship between velocity and particle flux. It is concluded that velocity exponents may be used only in very specific cases to identify erosion-corrosion mechanisms as the relationship between erosion-corrosion rate and velocity is complex and is a function of a wide range of parameters.
\end{abstract}

\section{Introduction.}

Wastage due to the combined effects of erosion and corrosion has become of increasing interest in recent years due to advances in coal-conversion processes, such as atmospheric and pressurized fluidized bed combustion. The advantage of these systems is improved thermal efficiency, which means that combustion temperatures can be lowered and, thus, $\mathrm{NO}_{x}$ and $\mathrm{SO}_{x}$ emissions reduced. In the early eighties, a severe wastage problem due to erosioncorrosion was identified for inbed heat exchanger tubes [1]. This led to the construction of laboratory rigs to simulate such wastage [2-4]. Low velocity erosion-corrosion research was carried out during that period [5] and the data from the various experimental rigs showed similarities $[2,4,5]$. The results from these investigations have shown that the extent of wastage is dependent on many parameters, including properties of the impacting particles, the target material, and the corrosive environment [6].

An important parameter in erosion-corrosion has been the velocity of the impacting particles. The general form of the equation relating erosion to velocity is given by:

$$
E=K . V^{n} f(\theta)
$$


where:

$$
\begin{aligned}
E & =\text { erosion rate; } \\
K & =\text { constant (function of particles and target); } \\
V & =\text { velocity; } \\
n & =\text { velocity exponent; } \\
\theta & =\text { impact angle. }
\end{aligned}
$$

Initially, models of erosion of ductile materials such as those of Finnie [7] and Hutchings [8], predicted a velocity exponent of 2. However, experimental work showed that the velocity exponent was nearer 2.5 than 2, and tended to increase with impact angle. Corrections to erosion models were later made to account for these factors $[9,10]$. For models of erosion of brittle material, the situation was less clear, with exponents derived which were higher than those for so-called "ductile" processes. Models which have been developed thus predict velocity exponents in excess of $3[11]$. However, very wide variations of velocity exponents have been reported for "brittle" erosion processes, ranging from 3 to 6 [12].

Frequently, investigators have attempted to use high velocity exponents as evidence for "corrosion-dominated" erosion-corrosion behaviour since wastage occurs by erosion of a brittle scale. However, there have been conflicting values of exponents derived for this regime, ranging from 0.8 to $7[2,3,13,14]$. Additional problems with comparing velocity exponents are variations in experimental rigs and in the erosion-corrosion parameters of the experiments.

This paper considers the effect of temperature, velocity, and alloy properties on the velocity exponent, in the different erosion-corrosion regimes. The main factors which may influence the evaluation of the velocity exponent are outlined and are discussed in relation to what is known at this stage about erosion-corrosion mechanisms.

\section{Effect of temperature on velocity exponent.}

The results to date have shown that the main factor influencing the value of the exponent, as the temperature is increased, is the relationship of particle flux with velocity. In the results reported by Sethi et al. [5], figure 1, the exponent derived at the lower temperatures, 2.18, was comparable to those reported for erosion-dominated "ductile" processes. As the temperature was increased, the exponent increased, up to 4.75 at $527^{\circ} \mathrm{C}$, and, subsequently, decreased to 4.19 at $557^{\circ} \mathrm{C}$. If the downward trend is extrapolated to higher temperatures, such as $627^{\circ} \mathrm{C}$, an exponent of 3.4 is derived. Hence, there is an increase in exponent with temperature during the transition from "erosion-dominated" to erosion-corrosion-dominated" behaviour, and a decrease in exponent with temperature once the transition temperature to "corrosiondominated" behaviour has been exceeded.

For the results reported by Ninham et al. [2] on the effect of velocity in a laboratory fluidized-bed apparatus, figure 2, the exponents (derived for wastage rate versus temperature) were significantly higher than those of the previous study. This is a good example of the effect of particle flux on the velocity exponent. In this apparatus, particle flux was dependent on velocity, and, thus, the exponents derived reflected the combined effects of velocity and flux on the increase in wastage; the values were higher than if flux were independent of velocity. The results showed no evidence of a decrease in the exponent derived at the higher temperature, although it is likely, given the downward trend in the curves, that a decrease 


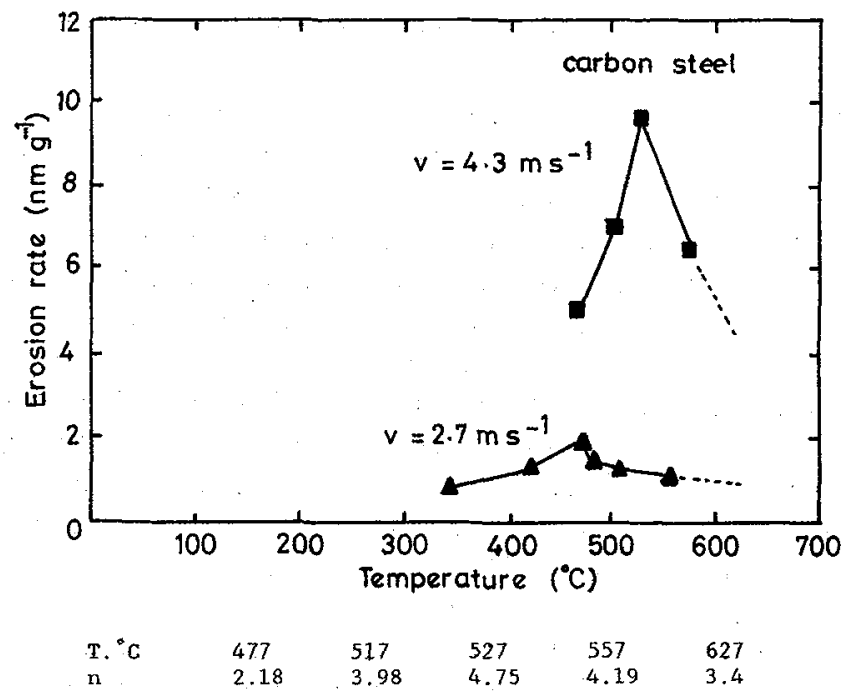

Fig. 1. - Erosion rate versus temperature for carbon steel at $30^{\circ}$ impact angles [5], showing the variation in velocity exponent $n$ with temperature. Erodent $1 \mathrm{~mm}$ alumina.

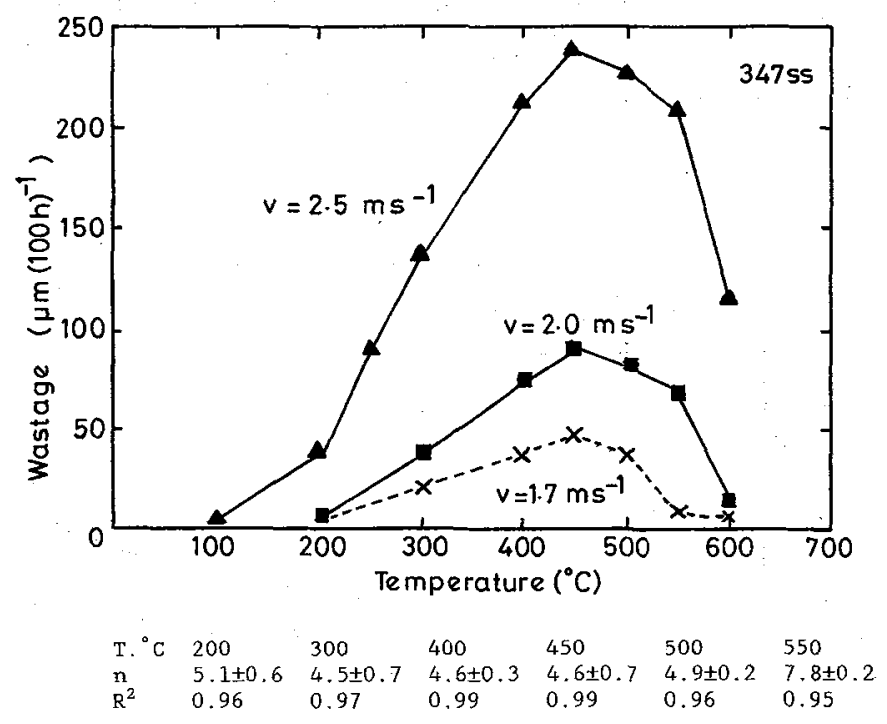

Fig. 2. - Wastage rate versus temperature for $347 \mathrm{SS}$ in a fluidized bed environment [2], showing the variation of $n$ with temperature. Erodent $170 \mu \mathrm{m}$ alumina.

would have been apparent, above $600^{\circ} \mathrm{C}$. Factors inherent to rigs may also affect the value of velocity exponent. Hence, the relative rather than the actual change of velocity exponent with temperature, may be a more meaningful indication of its relationship to temperature. 


\section{Variation of exponent as velocity is increased at elevated temperatures.}

The incubation period during which the erosion rate does not increase significantly with velocity at elevated temperatures, is the "corrosion-dominated" regime. A number of studies have been carried out to document this type of erosion behaviour, as velocity increases at high temperatures. Figure 3 is a plot of wastage against velocity for a nitrocarburized and asreceived 722M24T [15] alloy in a fluidized-bed environment at $500^{\circ} \mathrm{C}$. The graph shows that a transition in the relationship between wastage rate and velocity occurred at a critical velocity, and that this transition occurred at a higher velocity for the coated material. Evaluation of the exponents showed a number of trends, figure 3 . The overall average exponents, from $1.25-2.55 \mathrm{~ms}^{-1}$ for both coated and as-received material, were less than those in the higher part of the velocity range, $1.85-2.55 \mathrm{~ms}^{-1}$, and greater than those in the lower part of the range, at 1.25-1.75 ms $\mathrm{m}^{-1}$. Similar results have been found for the erosion of pre-oxidized and as received incoloy $800 \mathrm{H}$, figure 4 [16]. This indicates that there is a trend for the exponent to increase at the transition from "corrosion" to "erosion-corrosion-dominated" behaviour, which was a feature of the results on the effect of temperature, figure 1 . In addition, the exponents derived in the "corrosion-dominated" regime ranged between 1.0 to 1.7 , figures 3 and 4. These results are interesting in that are consistent with previous work in which velocity exponents in the range 0.9 to 1.8 were obtained for erosion in "corrosion-dominated" conditions at temperatures in excess of $500{ }^{\circ} \mathrm{C}$ [17-20]. These were surprising at that time as they were significantly lower than those evaluated for erosion of brittle materials at room temperature. This low exponent at high temperatures may be due to the enhanced plasticity of the scale at the higher temperatures causing the scale to exhibit deformation characteristics comparable to those of a metal. However, it is interesting that some of these exponents for the "corrosion-dominated" regime are lower than those derived for "ductile" processes at room temperature.

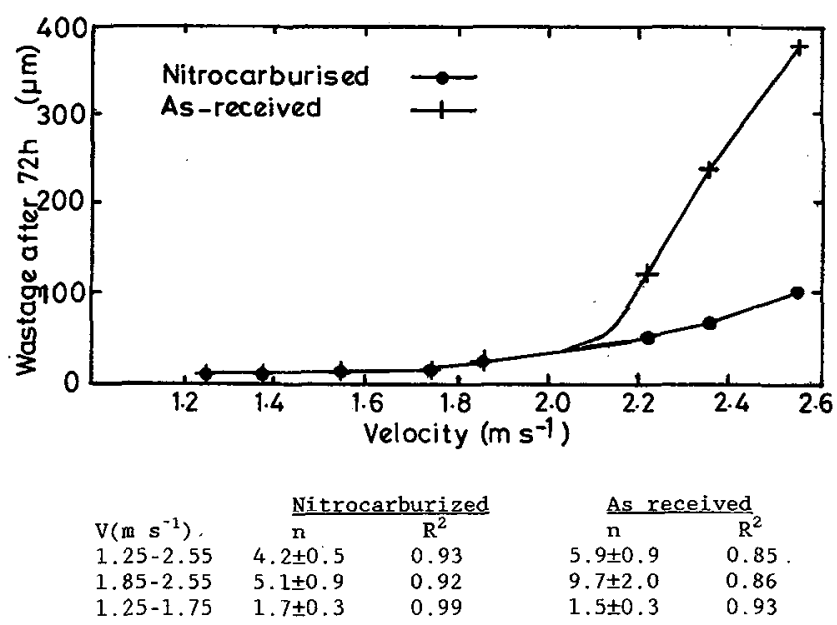

Fig. 3. - Wastage rate versus velocity for nitrocarburized and as-received $722 \mathrm{M} 24 \mathrm{~T}$ at $500{ }^{\circ} \mathrm{C}$ [15], showing the variation of $n$ for different ranges of velocity. Erodent $170 \mu \mathrm{m}$ alumina. 


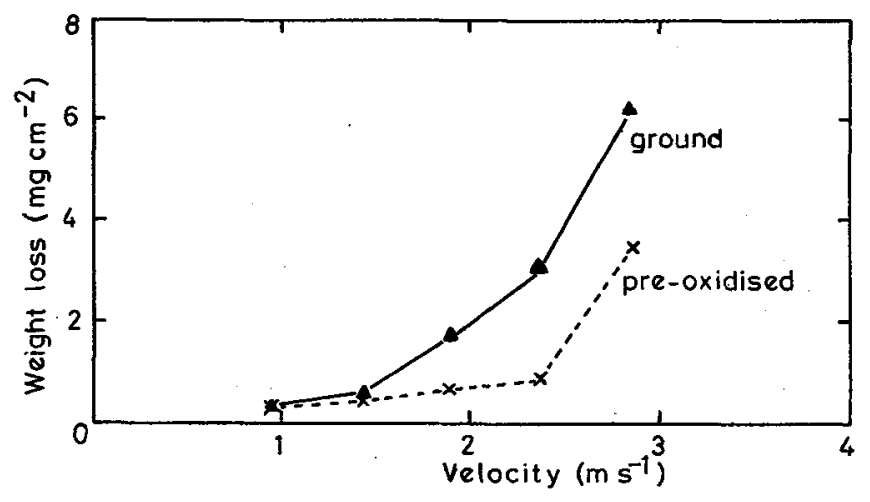

\begin{tabular}{lcccc} 
& \multicolumn{2}{c}{ Pre-oxidized } & \multicolumn{2}{c}{ Ground } \\
$\mathrm{V}\left(\mathrm{ms}^{-} \mathrm{l}\right)$ & $\mathrm{n}$ & $\mathrm{R}^{2}$ & $\mathrm{n}$ & $\mathrm{R}^{2}$ \\
$0.9-2.9$ & $2.0 \pm 0.6$ & 0.80 & $2.8 \pm 0.3$ & 0.98 \\
$0.9-2.4$ & $1.2 \pm 0.2$ & 0.95 & $2.6 \pm 0.3$ & 0.97 \\
$1.9-2.9$ & $4.0 \pm 1.7$ & 0.98 & $3.2 \pm 0.3$ & 0.99
\end{tabular}

Fig. 4. - Weight loss versus velocity for pre-oxidized and ground incoloy $800 \mathrm{H}$ at $500{ }^{\circ} \mathrm{C}$ [16], showing the variation of $n$ for different ranges of velocity. Erodent $200 \mu \mathrm{m}$ silica.

The results also show that coating or pre-oxidation of materials, figures 3 and 4, reduced the overall velocity exponent in the "erosion-corrosion-dominated" regime, as these pretreatments increased the critical velocity to erosion-corrosion of the underlying alloy. Other factors which may affect the incubation velocity and, hence the overall exponent are coating thickness and adhesion.

The value of the correlation coefficient associated with the exponent derived may give an arbitrary indication of whether the exponent describes either one or an amalgam of several erosion-corrosion regimes. For example, in figures 3 and 4, the correlation coefficients are lower for the overall exponent derived than for those derived at the lower and higher velocities, thus indicating a transition in wastage mechanism.

A puzzling feature of the results to date is that, the exponent was still high $(\approx 4)$, as the temperature for the transition to "corrosion-dominated" behaviour was attained, figures 1 and 2, whereas, the results on the effect of velocity, figures 3 and 4 show that the exponent was $\approx 1.5$ in the "corrosion-dominated" regime. Since the exponent tends to decrease as the temperature is increased further, figure 1 , it may suggest a re-examination of what has been classified as "corrosion-dominated" behaviour for the erosion rate versus temperature curves.

Hence, the results show that the exponents derived for erosion of ductile and brittle materials should only be used with certain reservations for modelling wastage in erosion-corrosion regimes. In the "erosion-dominated" regime, exponents derived for "ductile" erosion processes may be used in modelling this process. However, in the "erosion-corrosion-dominated" regime, the velocity exponents tend to exceed those in the "erosion-dominated" regime. The situation in the "corrosion-dominated" regime is more complex, with a trend for the exponent to tend to lower values than in the "erosion-corrosion-dominated", and "erosiondominated" regimes. This means that the common assumption of high velocity exponents in the "corrosion-dominated" regime is groundless. 


\section{Influence of alloy properties.}

Alloy composition determines oxidation resistance; thus, the velocity exponent derived at a given temperature will be a function of alloy composition, given the different affinities that alloy components have for oxygen. Figure 5 is a schematic diagram of the expected variation of wastage rate of two alloys of differing oxidation resistances (oxidation resistance of alloy $1<$ alloy 2), at two velocities $\left(v_{1}<v_{2}\right)$. This schematic is sketched on the basis of experimental results [5,6]. It can be seen that, at temperature $T_{1}$, the exponent is greater for the alloy with lower oxidation resistance, alloy 1 , than for alloy 2 . However, as the temperature is increased to $T_{2}$, and the transition to "corrosion-dominated" behaviour occurs for alloy 1 at $v_{2}$, the situation reverses with the exponent for alloy 2 being higher than that for alloy 1 . Some of the earlier results reported in the laboratory fluidized bed environment [21] showed that the ferritic steel 410 was more sensitive to velocity than austenitic steel 310 at $500{ }^{\circ} \mathrm{C}$. This may have been due to the differences in the oxidation resistances of the alloys at the higher temperatures.

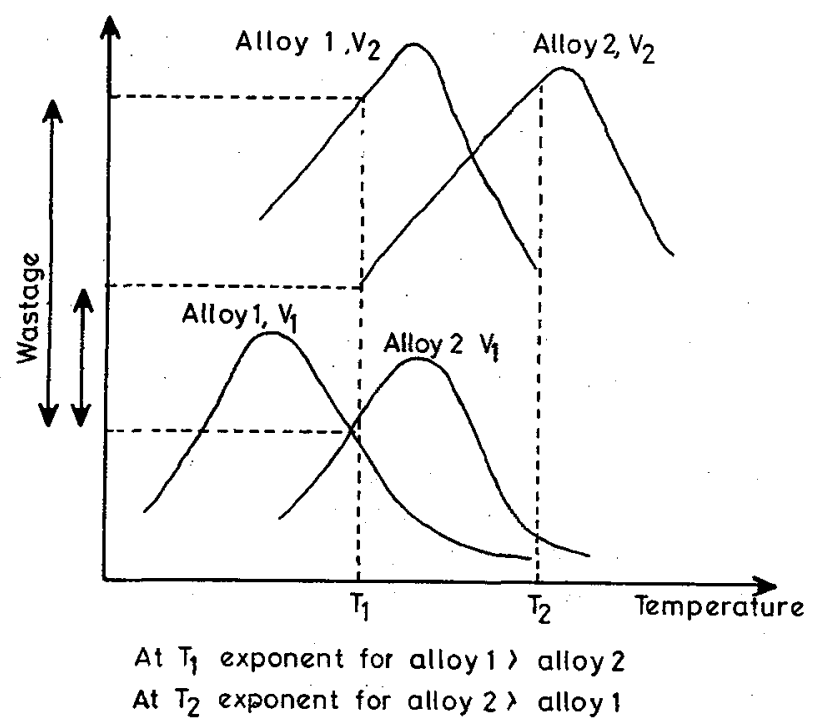

Fig. 5. - Schematic diagram of the expected variation of alloy wastage with temperature for two alloys of different oxidation resistances, and for two velocities. (Oxidation resistance of alloy $1<$ than that for alloy 2). $\left(v_{1}<v_{2}\right)$.

Properties of the oxide scale may affect the velocity exponent. A poorly adherent oxide may be more sensitive to effects of velocity than a protective adherent scale. Increasing oxide ductility should increase the critical velocity to damage of the scale and hence result in a decrease of the exponent derived. The growth rate of the oxide will also affect the velocity exponents, as shown in figure 5 .

Alloy properties, such as yield strength, may also influence the velocity exponent, particularly at higher temperatures, where a reduction in yield strength occurs. If erosion is the dominant process at higher temperatures, the velocity exponent may be higher than at room 
temperature. Since there is no correlation between hardness of steels and erosion resistance at room temperature [22], conventional microstructural hardening mechanisms should have little effect on velocity exponents derived for wastage in low temperature "erosion-dominated" conditions.

Evaluation of the velocity exponent in the stricter sense requires knowledge of the $K$ values in equation (1) for the various erosion-corrosion regimes. Since these are difficult to estimate for the "erosion-corrosion" and "corrosion-dominated" regimes, the $K$ values are generally assumed to be equal. This means that if a transition in erosion-corrosion regime occurs, as shown in figures 3 and 4 , the exponent will reflect the increase or decrease in the $K$ value, which has not been accounted for in the evaluation. This is an important reason why it may be erroneous to compare velocity exponents in erosion-corrosion regimes to those derived for solid particle erosion processes at room temperature.

\section{Conclusions.}

i) There is a wide variation of velocity exponents derived for different erosion-corrosion conditions, which reflect the differences in erosion resistance of the scale formed between impacts.

ii) Exponents derived for erosion of alloys in so-called "erosion-dominated" conditions, can be correlated to those derived for "ductile" erosion processes. However, those for the "erosion-corrosion-dominated" regime are higher than for the "erosion-dominated" regimes.

iii) As the transition to "corrosion-dominated" behaviour occurs, the velocity exponents tend to values which are significantly lower than those for the other erosion-corrosion regimes, and for solid particle "brittle" erosion processes.

\section{References}

[1] MEADOWCROFT D.B., Mat. Sci. Eng. A 121 (1989) 669.

[2] Ninham A.J., Hutchings I.M., Little J.A., Proc. Conf. Corrosion '89 (NACE, Houston, 1989).

[3] StotT F.H., Stack M.M., Wood G.C., Proc. Corrosion-Erosion-Wear of Materials at Elevated Temperatures, Vol. 12 (NACE, Houston, 1990) p. 1-16.

[4] MAC AdAm S.S., Stringer J., Proc. Workshop on Wastage of In-bed Surfaces in Fluidized Bed Combustors, paper 5.8 (EPRI, 1987).

[5] SeTHI V.K., CoReY R.G., Proc. 7th Int. Conf. on Erosion by Liquid and Solid Impact (Cambridge, 1987) p. 73.

[6] STACK M.M., STOTT F.H., Wood G.C., Materials for Combined Cycle Power Plant, Pub. Inst. Mater. U.K. (1991) 253.

[7] FinNie I., Proc. U.S. Nat. Cong. App. Mech. (1958) 527.

[8] Hutchings I.M., Proc. 5th Int. Conf. on Erosion by Liquid and Solid Impact (Cambridge, 1979) p. 36.

[9] FINNIE I., MAC FADDEN D.H., Wear 48 (1978) 181.

[10] Hutchings I.M., Wear 70 (1981) 269.

[11] Evans A.G., Gulden M.E., Rosenblatt W.H.E., Proc. R. Soc. A 361 (1978) 343. 
[12] RUfF A.W., WIEDERHORN S.M., Erosion by solid particle impact, Treatise in Mater. Sci. Tech 16, Mater. Erosion.(Acad. Press, 1979).

[13] Wright I.G., NaGarajan V., StRinger J., Oxid. Met. 25 (1986) 175.

[14] Levy A.V., Wang BuQuian, Yong Fa Man, JeE Nancy, Proc. 7th Int. Conf. on Erosion by Liquid and Solid Impact (Cambridge, 1987) p. 72.

[15] Rogers P.M., HuTChings I.M., LiTTLE J.A., Surface Engineering, S.A. Meguid Ed. (Pub. Elsevier, 1990) in press.

[16] STACK M.M., STOTT F.H., WoOd G.C., Mater. Sci. Tech. 7 (1991) 1128.

[17] TABAKOFF W., VitTAL B.V.R., Wear 86 (1983) 89.

[18] Gat N., Tabakoff W., J. Test. Eval. 8 (1980) 177.

[19] ZhOU J., BhaduR S., Corrosion and Particle Erosion at High Temperatures (Pub. Mineral, Metals and Materials Society, 1989) p. 315.

[20] LEVY A.V., YONG Fa Man, Wear 98 (1989) 141.

[21] Ninham A.J., Entwisle M.J., Hutchings I.M., Little J.A., Proc. 10th Int. Conf. on FBC (1989)

[22] FINNIE I., WOLAK J., KABIL Y.H., J. Mater, 12 (1967) 682. 